\title{
A comparative study of boar semen extenders with different proposed preservation times and their effect on semen quality and fertility
}

\author{
Marina Anastasia Karageorgiou, Georgios Tsousis, Constantin M. Boscos, Eleni D. Tzika, \\ Panagiotis D. Tassis, Ioannis A. Tsakmakidis
}

Aristotle University of Thessaloniki, School of Veterinary Medicine, Faculty of Health Sciences, Thessaloniki, Greece

Received May 19, 2015

Accepted February 10, 2016

\begin{abstract}
The present study compared the quality characteristics of boar semen diluted with three extenders of different proposed preservation times (short-term, medium-term and long-term). A part of extended semen was used for artificial insemination on the farm (30 sows/extender), while the remaining part was stored for three days $\left(16-18{ }^{\circ} \mathrm{C}\right)$. Stored and used semen was also laboratory assessed at insemination time, on days 1 and 2 after the collection (day 0 ). The long-term extender was used for a short time, within 2 days from semen collection, with the aim to investigate a possible advantage over the others regarding laboratory or farm fertility indicators at the beginning of the preservation time. Viability, motility, kinetic indicators, morphology and DNA fragmentation were estimated. The results showed reduced viability, higher values for most of the kinetics, and higher immotile spermatozoa from day 1 to day 2 in all extenders; however, the long-term extender was superior compared to the other two on both days. With regard to morphology and chromatin integrity, the percentage of abnormal and fragmented spermatozoa increased on day 2 compared to day 1 for all of the extenders. However, based on the farrowing rate and the number of piglets born alive after the application of conventional artificial insemination within 2 days from semen collection/dilution, it was found that the medium-term diluents were more effective. In conclusion, it seems that the in vivo fertilization process involves more factors than simply the quality of laboratory evaluated sperm indicators, warranting further research.
\end{abstract}

DNA fragmentation, ejaculate, in vitro quality, in vivo fertility, semen analysis, semen storage

Conventional examination of boar sperm on commercial farms aims to evaluate the fertilizing capacity and to control the conditions of breeding and sperm manipulation after the collection of the ejaculate (Tsakmakidis et al. 2010). Semen quality characteristics are affected by many factors, including the type of semen extender, storage duration and temperature (Fraser and Strzezek 2004).

Boar semen diluents are classified by the commercial providers into three categories: short-term (ST), medium-term (MT) and long term (LT) extenders according to their preservation capacity (1-2, 3-4 and 7-10 days after collection, respectively). Long-term extenders allow for long distance sperm transportation. Moreover, the acquired time flexibility enables collection centres to conduct specialized diagnostic tests and to better organize semen procedures (Gadea 2003).

In literature, only few studies compare the preservation capacity of different extenders in the laboratory and the relation with actual field conditions. To our knowledge, data on the efficacy of swine artificial insemination (AI) 1-2 days from collection and dilution in long-term extenders are even scarcer. This category of extenders is supposed to maintain sperm quality within acceptable levels (i.e. total sperm number $>35 \times 10^{9}$ sperm/ejaculate, gross motility $>70 \%$, abnormal morphology $<20-25 \%$ ), for a long period of time (for 5-7 or up to 10 days) and their use is indicated when insemination must occur beyond the

Address for correspondence:

Ioannis A. Tsakmakidis

Faculty of Health Sciences

School of Veterinary Medicine

Aristotle University of Thessaloniki, Greece
Phone: +302310994467

E-mail: iat@vet.auth.g

http://actavet.vfu.cz/ 
preservation time achieved with the short-term and medium-term extenders. However, it is unclear whether LT extenders have an advantage over the others regarding laboratory or fertility indicators at the beginning of the preservation time. If the long-term extenders combined the desirable in vitro and in vivo sperm quality during the days of the classic insemination protocol, their use could disengage breeders from the strict keeping of times, accomplishing the preservation of sperm for a minimum or maximum time according to the needs of each farm. The aim of this study was to investigate the potential in vivo and in vitro superiority of LT, compared to ST and MT extenders, after their use for a shorter time than recommended by the technical specifics. For that purpose, the effects of three extenders with different proposed preservation time (short-term, medium-term and long-term), were examined comparatively both in laboratory evaluated sperm quality and in vivo fertility, after the preservation of sperm for three days.

\section{Materials and Methods}

\section{Boar semen extenders}

Aiming to focus on different preservation term extenders' effect and minimize the possible effect of different commercial extenders' composition, all of the extenders used came from the same commercial supplier (Magapor ${ }^{\circledR}$, Spain). The products BIOPIG, OPTIM-I.A. and DURAGEN were used as short-term, medium-term and long-term extenders, respectively. The composition of the extenders was not provided by the company.

Sperm collection-dilution and application of artificial insemination

Semen samples were collected from 5 adult (2-3 years old) crossbred boars of a 700 capacity pig farm in northern Greece. A total of 20 ejaculates (4 per boar) were obtained during the period of five months (FebruaryJune). Two hours before semen collection, the selected extenders (short-term, medium-term and long-term) were prepared according to the instructions of the supplier company. The "sperm rich fraction" was collected by an experienced farm employee by the gloved-hand technique, gel was separated by a gauze and raw semen was transported to the farm laboratory in an isothermal glass vessel $\left(37^{\circ} \mathrm{C}\right)$. Initially, vitality, morphology and motility of the sperm were microscopically assessed, followed by the calculation of density by the use of a photometer (SDM1, Minitube, Germany). Ejaculates of acceptable quality (volume $>200 \mathrm{ml}$, concentration $>200 \times 10^{6}$ spermatozoa/ml, total sperm number $>40 \times 10^{9}$ sperm/ejaculate, gross motility $>70 \%$, abnormal morphology $<20-25 \%$ ) were more processed. Each ejaculate was divided into 3 equal fractions in terms of volume and each part was diluted with one of the 3 different extenders at a temperature of $30{ }^{\circ} \mathrm{C}$ until a final concentration of $30 \times 10^{6} \mathrm{spermatozoa} / \mathrm{ml}$. The final product was packaged in vials of insemination with the volume of $80 \mathrm{ml}$. On collection day (day 0) the insemination doses of semen with each of the three different diluents were stored at controlled temperature conditions $\left(16-18^{\circ} \mathrm{C}\right)$ up to the performance of classical AI in the farm. Aiming to perform the special laboratory semen evaluation tests, diluted semen doses were transferred within 30 min and stored at the laboratory of biotechnology of reproduction in the Clinic of Farm Animals, School of Veterinary Medicine, Thessaloniki. The transportation and storage of semen was performed in an isothermal box equipped with an alarm sensor to ensure the appropriate temperature $\left(16-18^{\circ} \mathrm{C}\right)$ during storage. At collection and insemination dates semen aliquots from each dose were laboratory assessed and directly transferred to the pig farm for the performance of artificial insemination. Therefore, the ejaculates that were used for artificial insemination in the field study, were also evaluated by laboratory assessments. Sows were checked for the onset of oestrus every $24 \mathrm{~h}$ using a boar detector. Those who exhibited oestrus were classified weekly into three homogenous groups (30 sows per group) based on their age (between 20 and 36 months old) and the number of their previous births $(\geq 2)$. Insemination was applied according to pig farm's routine, at the time of the first oestrus detection (day 1) and $24 \mathrm{~h}$ later (day 2), using the same boar semen donor, diluted with one of the three extenders. During insemination the sows received boar exposure to minimize semen loss and back-flow (Willenburg et al. 2003). The three sow groups were monitored for return of oestrus, and the farrowing rate and number of live born piglets were recorded.

Laboratory semen evaluation

The following reagents were used:

Eosin (Eosin, 32617, Riedel-de Haen ${ }^{\circledR}$, Sigma Aldrich, Seelze, Germany)

- Nigrosine (Nigrosin, 8005-03-6, Aldrich $^{\circledR}$, Milwaukee, USA)

- Acridine orange (Acridine orange, A6014, Sigma Aldrich ${ }^{\circledR}$, Seelze, Germany)

- Spermblue (SpermBlue, 08029, Mcroptic SL ${ }^{\circledR}$, Automatic Diagnostic Systems, Barcelona, Spain)

The following instrumentation was used:

- Automatic Analyser: Sperm Class Analyser ${ }^{\sqrt{ }}$, Microptic SL, Automatic Diagnostic Systems, Barcelona, Spain

- Microscope: Microscope ZEISS, AXIO, Scope A1, Germany

- Fluorescence microscope: Olympus BX 41, Japan 
Assessments

Diluted semen samples were maintained for 3 days at $16-18{ }^{\circ} \mathrm{C}$ (collection day 0 , storage days 1,2 ). On the days of storage, $1.5 \mathrm{ml}$ of sperm diluted with each one of the three extenders was heated in Eppendorf vials in water bath at $37^{\circ} \mathrm{C}$, in order to proceed to the estimation of motility, morphology, viability and chromatin integrity assay.

Motility was estimated with the use of Computer Assisted Semen Analysis (CASA).

The assessed indicators were:

- Immotile spermatozoa \%

- Progressive spermatozoa \%

- Rapid, medium and slow spermatozoa $\%(10<$ slow $<25<$ medium $<45<$ rapid $\mu \mathrm{m} / \mathrm{s})$

- Curvilinear velocity-VCL $(\mu \mathrm{m} / \mathrm{s})$, straight line velocity-VSL $(\mu \mathrm{m} / \mathrm{s})$, average path velocity-VAP $(\mu \mathrm{m} / \mathrm{s})$, amplitude of lateral head displacement-ALH $(\mu \mathrm{m})$, beat/cross-frequency-BCF $(\mathrm{Hz})$, straightness-STR $($ VSL/VAP $\times 100)$, linearity-LIN $($ VSL/VCL $\times 100)$, wobble-WOB $($ VAP/VCL $\times 100)$

- Hyperactivation (sperm subpopulation of increased VCL $>97 \mu \mathrm{m} / \mathrm{s}, \mathrm{ALH}>3.5 \mu \mathrm{m}$ and decreased LIN < 0.32)

CASA was configured as follows: 10 fields and $>500$ spermatozoa, 25 frames/s, region of particle control 10-18 microns, progressive movement of $>45 \%$ of the indicator STR, circumferential movement $<50 \%$ LIN, depth of field 10 microns and temperature of the microscope plate at $37^{\circ} \mathrm{C}$. In order to assess the motility of each sample, pictures of at least 4 fields were taken $(\times 100)$, in order to record the movements of 500 spermatozoa or more. The number of objects incorrectly identified as spermatozoa was manually removed and final analysis was done for each sample. Analysis was performed by Sperm Class Analyzer software (SCA v.3, Microptic S.L., Spain).

Morphology was estimated by the SpermBlue staining method, taking into account the instructions of the manufacturer. The assessment was realized by microscopic observation (magnification $\times 400$ ). Spermatozoa were classified into two categories: a) normal, b) with morphological abnormalities (head, neck, tail, protoplasmic droplets). Finally, 200 spermatozoa were scored and the \% ratio per sample was calculated.

Viability was estimated with the use of double staining eosin-nigrosine in one step (World Health Organization 2010). By means of an optical microscope (magnification $\times 1000) 200$ spermatozoa were estimated and counted, while the results were expressed in $\%$ ratio.

DNA integrity was estimated with the use of Acridine Orange Test (AOT) (Tejada et al. 1984). The final assessment took place in a dark room, using an optical fluorescence microscope (Olympus BX 41, Japan) equipped with a digital camera and image analyser computer software (U-TV 0.35 C-2, Imaging Software System GmbH for Windows, Olympus, Japan) at $\times 1000$ magnification. Ten different fields were counted and estimated, while 200 spermatozoa were scored. The results were expressed in a \% ratio.

The statistical analysis was performed using the Statistical Analysis Systems version 9.0 (SAS Institute Inc., 1996, Cary, N.C., U.S.A.). Sample size was approximately estimated by power analysis (power $\geq 85 \%$ ) for one-way ANOVA with the use of the Analyst Application of SAS. All data were tested for normality before analysis. Indicators "Immotile, Progressive, Rapid, Medium, Slow, VCL, VSL, VAP, LIN, STR, WOB, ALH, BCF" followed a normal distribution. Indicators "Hyperactivation, Normal, Abnormal, Viability, DNA" were normalized by square root transformation. The least square means were analysed with the use of a General Linear Mixed Model (PROC MIXED). The model included the type of extender (short-term, medium-term, long-term) and time ( $1^{\text {st }}$ and $2^{\text {nd }}$ day) and their interactions as fixed effects; and included boar and number of ejaculation nested within boar and time as random effects. Least-squares means were obtained from each class of the factors; and were compared by using least significant different test (LSD) with Tukey-Kramer adjustment for multiple comparisons. The same statistical analysis was used to compare live litter size including the type of extender, boar and their interaction. Pregnancy rates between types of extenders were compared using the chi-square and the fisher's exact test (PROC FREQ). Data were presented as mean \pm relative standard deviation (RSD). Significant difference was defined as $P<0.05$.

\section{Results}

The percentage of immotile spermatozoa increased significantly on day 2 in ST and MT diluents, but not in LT (Table 1). Thus, on day 2 the aforementioned indicator was significantly higher for the ST compared to the LT extender and of the same magnitude as the MT extender. Regarding the percentage of spermatozoa with strong progressive movement, a significant reduction on day 2 was evident only for the ST extender. However, on day 2, the LT extender showed higher values than both ST and MT extenders. The percentage of rapidly moving spermatozoa decreased significantly over time only in the MT diluents, whereas on day 2, the LT extender was again superior to the MT extender and numerically also corresponded to ST. Finally, there were no significant differences between 
the 3 extenders regarding the percentage of slow moving and hyper-activated spermatozoa on either day.

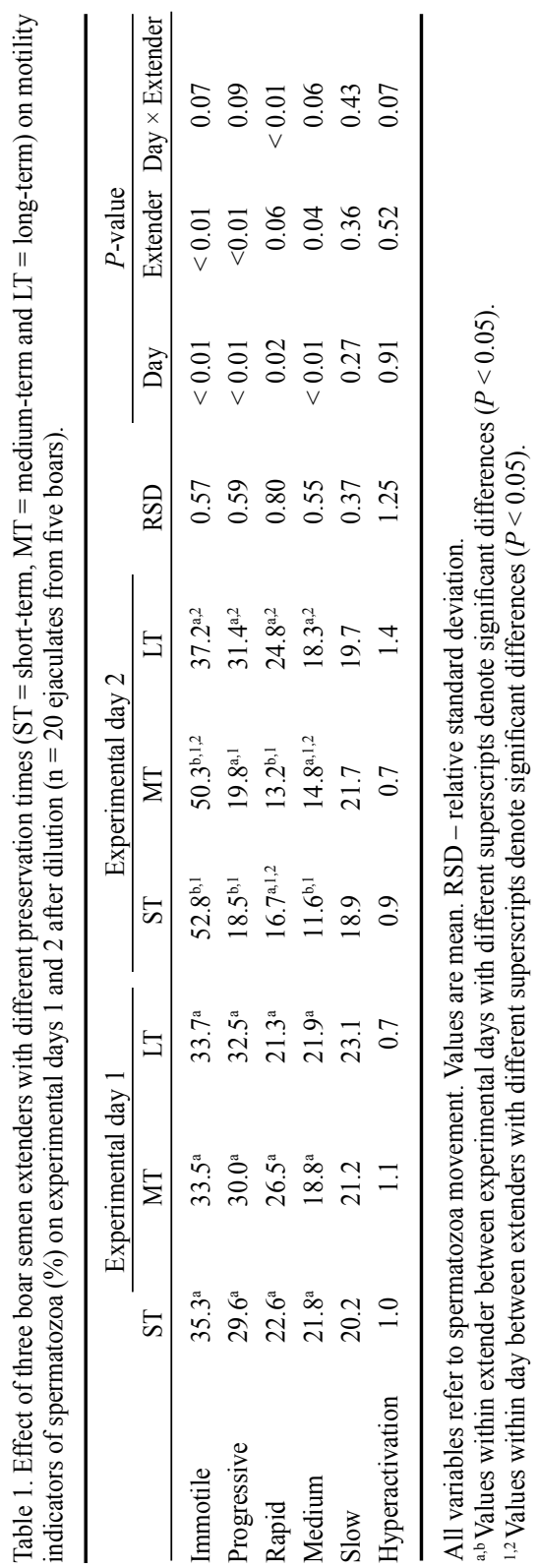

The values of the individual kinetic indicators of VCL, VSL and VAP are shown in Table 2. Regarding time, a significant decrease was observed only in the medium-term extender. Moreover, the LT extender predominated over the others. The WOB kinetic indicator was significantly higher on day 2 for the LT extender compared to the ST extender. The remaining kinetic indicators (LIN, STR, ALH, BCF) did not change significantly with time.

The percentage of morphologically normal spermatozoa decreased over time in ST and LT diluents, but not in the MT diluent (Table 3). Sperm viability was reduced with time in all the examined extenders and it was significantly different between the three extenders on both days, with the LT extender showing the highest values followed by the MT and ST extenders. Regarding DNA fragmentation, it was significantly increased on day 2 compared to day 1 in all the diluent groups. However, no significant differences were observed among the extender groups on both tested days.

Field fertility records are listed in Table 4. No significant differences were observed between MT and LT extender groups. However, the farrowing rate and the number of live born piglets were significantly lower in the ST extender group compared to the MT extender group.

\section{Discussion}

In the present study, we compared three extenders with different proposed preservation capacities with regard to various laboratory indicators and the fertilizing ability after conventional AI on the farm. The in vitro sperm quality was evaluated by motility, morphology, viability and sperm chromatin integrity determination, while the in vivo fertility was assessed by recording the farrowing rate and the number of live born piglets. Sperm motility is an important indicator for the evaluation of sperm quality, as it implies an active metabolism, integrity of membranes and is positively correlated with the fertilizing capacity. In the present trial, sperm diluted in the long-term extender maintained its quality at an acceptable level, for the 3 days of preservation. This partly confirms the study of Kaeoket et al. (2010), who found that diluents can satisfactorily maintain the motility of boar sperm at 


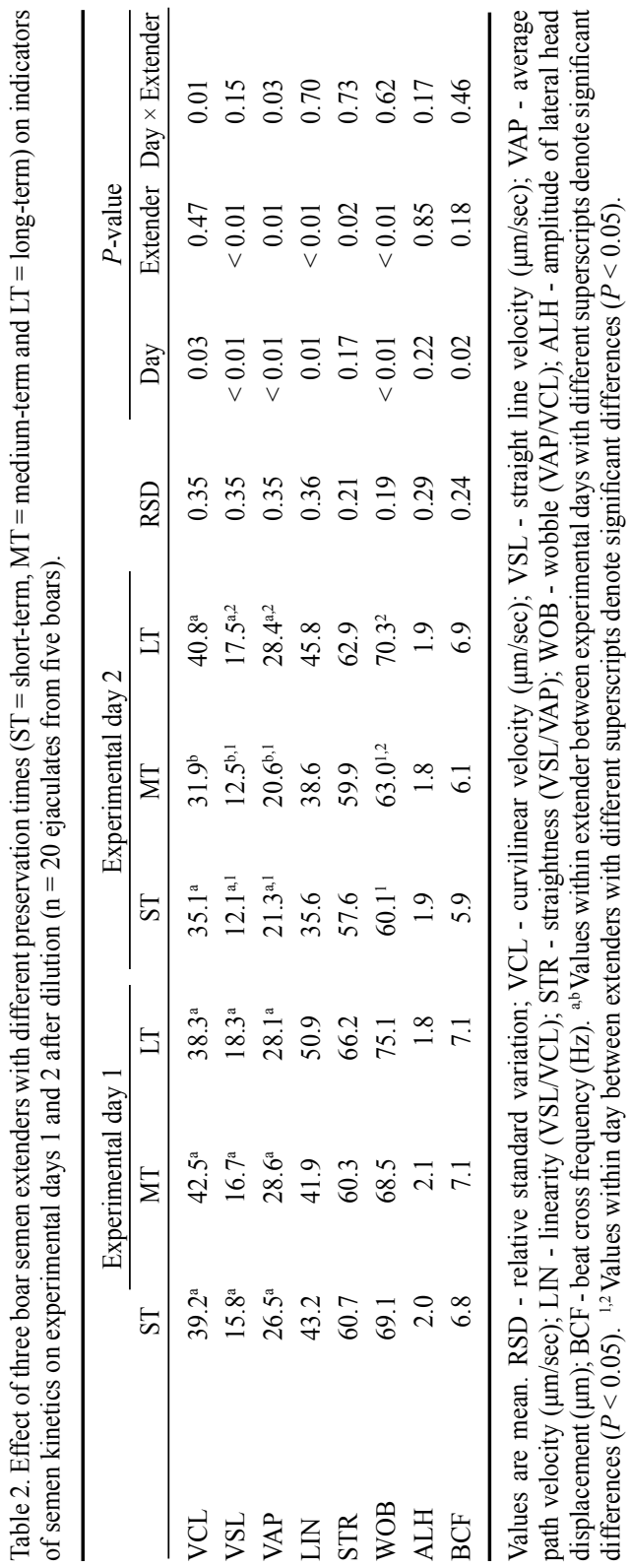

$18{ }^{\circ} \mathrm{C}$ for 8 days. However, in our study some differences in kinetic indicators were noticed after 3 days of storage. Specifically, VCL, VSL and VAP significantly decreased in the mediumterm extender, in contrast with the long-term one, which remained almost stable. Moreover, Estienne et al. (2007) reached a similar conclusion, after a computer assisted sperm analysis of motility at seven days of storage at $18{ }^{\circ} \mathrm{C}$. In contrast, with the addition of LIN, the aforementioned indicators were not affected in the study of De Ambrogi et al. (2006) either by the category of the diluent used (short-term and medium-term) or by preservation time $\left(96 \mathrm{~h}\right.$ at $\left.17^{\circ} \mathrm{C}\right)$. It is noteworthy that in our study, the LIN indicator also remained unaffected. From the evaluation of various forms of motion, it was also found that for the long-term extender the number of rapidly moving spermatozoa was increased on the $2^{\text {nd }}$ day of the experiment compared to the $1^{\text {st }}$ day. The number of spermatozoa moving at low and medium speed decreased depending on time, but the changes were not significant. The increased sperm motility in the long-term medium compared to the short-term one, was also the conclusion of a study, where frozenthawed boar semen was used (Kaeoket et al. 2011).

In the present study, viability was decreased in all three extender groups, a result that is consistent with the reduction of sperm motility. This outcome is in agreement with the findings of Frydrychova et al. (2010) who used only long-term means. It should be noted that in our study, the percentage of live spermatozoa was higher than the one of motile ones (i.e. some of the

live spermatozoa did not move). A possible dysfunction of cell mitochondria could be inducing loss of motility in spermatozoa with intact membranes. The semen samples that were diluted in the long-term extender had a higher viability for the entire duration of the trial, compared to the samples of the short-term and medium-term extender groups. The higher percentage of live spermatozoa on the $2^{\text {nd }}$ day of the experiment was expected for long-term diluents according to the manufacturer's specifications. Kaeoket et al. (2011) studied the quality of frozen-thawed boar semen after its dilution in short-term and long- 


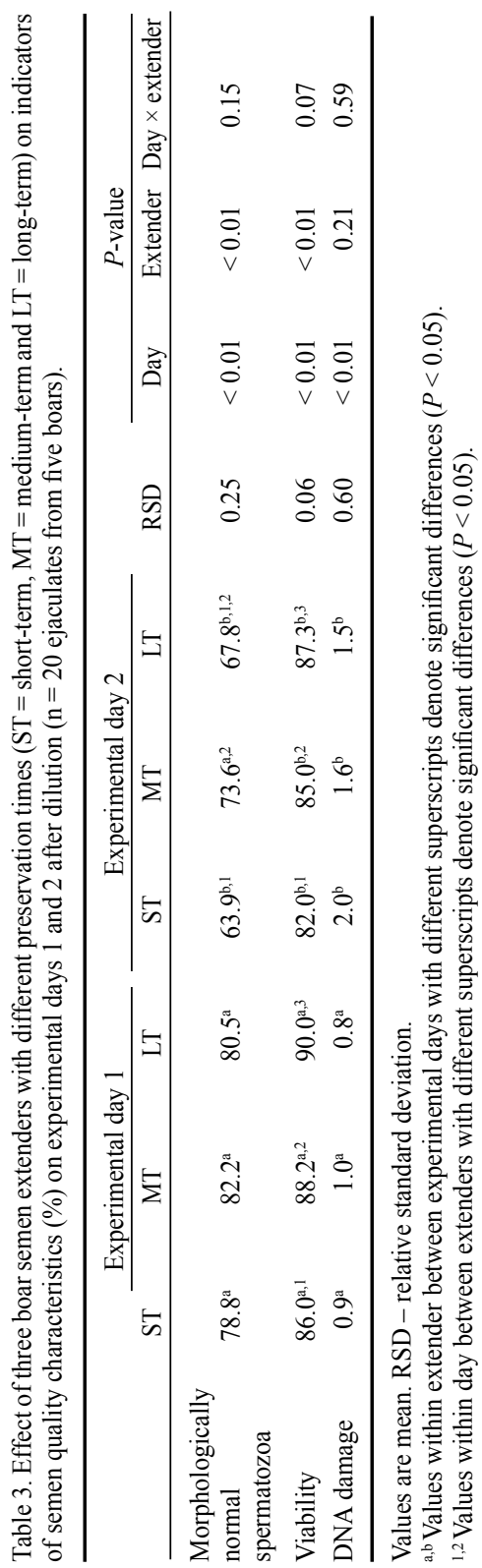

term extenders and reached a similar conclusion regarding viability. However, Vyt et al. (2004) compared the maintenance capacity of long-term and short-term extenders for a seven-day period and found no significant differences in the percentage of dead spermatozoa. In addition, De Ambrogi et al. (2006) studied sperm preservation using the same diluents at $17{ }^{\circ} \mathrm{C}$ for $96 \mathrm{~h}$ and indicated that the kind of extender had no effect on the viability of spermatozoa.

Morphological abnormalities, depending on their position, affect the movement and potentially the fertilizing ability of the gametes. Taking into account Maes et al. (2011), at least 70\% of morphologically normal spermatozoa per insemination dose are required to achieve high fertilization rates. However, Vyt et al. (2008), reported that morphological characteristics have a small predictive value of sperm fertility. In our study, the percentage of normal spermatozoa was significantly reduced in all extenders during preservation. The highest percentage of normal cells was observed in medium-term extender samples, both on the $1^{\text {st }}$ and $2^{\text {nd }}$ day of the trial. Probably, this result, combined with the higher in vivo efficiency of the medium-term extender supports the necessity of sperm morphology evaluation.

In recent years, the integrity of sperm DNA has been evaluated as an additional factor associated with fertility. Some researchers have positively correlated the integrity of sperm chromatin with other qualitative semen variables such as motility and morphology (Foxcroft et al. 2008). Others, such as Perez-Llano et al. (2006) found that DNA fragmentation is negatively correlated with the evaluation of classic sperm variables. However, both studies agree that it is affected by semen handling and is associated with fertility and embryo development efficiency. In our study, a significant reduction of the integrity of chromatin was found on the $2^{\text {nd }}$ day compared to the $1^{\text {st }}$ day of the experiment for all diluents. This finding reinforces the results of Boe-Hansen et al. (2005) who found that after $72 \mathrm{~h}$ of sperm storage the integrity of sperm DNA chromatin was significantly reduced. In contrast, De Ambrogi et al. (2006) did not reveal any difference in the integrity of the sperm chromatin associated with different diluents or preservation time after sperm storage for $96 \mathrm{~h}$ at $17^{\circ} \mathrm{C}$.

The relation between in vitro sperm quality characteristics and field fertility is an issue studied in various animal species. Vyt et al. (2008) concluded that the individual kinetic indicators, when studied one by one, do not lead into significant results, compared with the study of motility as an indicator itself. They also mentioned that motility is significantly 
associated with the litter size and the number of live born piglets. Tardif et al. (1999) also referred a positive relation of motility with the number of live born piglets. In the present study, although semen motility in the long-term extender group was higher than in the other two groups, the highest rate of farrowing and number of live born piglets was achieved with semen of the medium-term extender group. Although, the latter was not significantly different from the long-term extender group, it is indicative of other factors that affect the fertility process into the female genital tract. There is a probability that the contractility of the uterus, which normally increases during oestrus, is stimulated to such an extent that sperm movement is impaired in the genital tract and the chance of rejection/regression increases (Langendijk et al. 2005). Non-massive activation of accumulated sperm in the sperm reservoir of the oviducts is mentioned as another factor that can affect fertilization (Rodriguez-Martinez et al. 2005). Sperm must survive within the female genital tract, without possessing the recovery mechanisms of other types of cells. Moreover, for the female body spermatozoa are foreign cells and they may be confronted with the defence mechanisms of the immune system (Suarez and Pacey 2006). Taking into account Gadea et al. (2004) the evaluation of sperm quality indicators contributes to the identification of those who have low fertilizing capacity, but it cannot effectively predict the in vivo fertility. Furthermore, according to Kommisrud et al. (2002) sperm motility is an important quality indicator, but it does not assure by itself the fertilization ability of sperm.

Table 4. Field fertility data after the use of lab evaluated stored boar semen diluted with extenders with different preservation times $(\mathrm{ST}=$ short-term, $\mathrm{MT}=$ medium-term and $\mathrm{LT}=$ long-term $)(\mathrm{n}=90$ inseminated sows, 30 per tested extender).

\begin{tabular}{lcccc}
\hline & ST & MT & LT & RSD \\
\hline Farrowing rate \% & $56.7^{\mathrm{a}}$ & $86.7^{\mathrm{b}}$ & $76.7^{\mathrm{a}, \mathrm{b}}$ & \\
Number of live born piglets & $10.3^{\mathrm{a}}$ & $12.7^{\mathrm{b}}$ & $11.2^{\mathrm{a}, \mathrm{b}}$ & 0.1 \\
\hline
\end{tabular}

Values are mean. RSD - relative standard deviation.

${ }^{\mathrm{a}, \mathrm{b}}$ Values within row with different superscripts denote significant differences $(P<0.05)$.

Generally, selection of appropriate diluents should always aim to maximize the farrowing rate and the number of live born piglets, taking into account the particularities of each farm. Poor management of animal breeding can have adverse economic effects (Gadea 2003). According to the findings of this study, the use of medium-term extenders which is most prevalent nowadays appears to be advantageous when applied according to the classical protocol of AI which includes the use of sperm within 1-2 days after collection. The short-term extender revealed poor fertility results in this study probably because of its use on the limits of its efficiency ( 1 and 2 days after collection day). On pig farms where semen is primarily self-produced and the interval between collection and inseminations is very small, medium-term extenders are sufficient to maintain sperm quality and are cheaper than the long-term ones. Concerning AI performance, Haugan et al. (2007) found similar fertility rates after doubly applied insemination with diluted semen in shortterm and long-term means. Kuster and Althouse (1999) assessed the fertility of boar semen after its preservation for 2-6 days in two different long-term means and found no significant reduction of fertility and litter size up to the $6^{\text {th }}$ day of the study for one of the two extenders. It is obvious that boar semen dilution and preservation is a promising scientific area. The in vivo long-term adequacy of extenders beyond 2 to 3 days of the classical protocol of artificial insemination needs further study. It is possible that long-term extenders are able to maintain sperm fertility for a longer period and be advantageous over other types of diluents. 
In conclusion, long-term extenders exhibit greater efficiency maintaing in vitro quality of boar semen, after a 3-day preservation of boar sperm at $17^{\circ} \mathrm{C}$ compared to mediumterm and short-term ones. However, insemination within two days after collection with semen diluted and stored using a medium-term extender leads to a numerically higher farrowing rate and number of live born piglets compared to a long-term extender, and to significantly higher farm records compared to a short-term extender. Therefore, classical semen quality indicators are not the only ones affecting the complex procedure of the in vivo fertilization. Further investigation of the field conditions and the genetic material of sows and boars could fully respond to questions of this particular scientific field.

\section{Acknowledgements}

This trial was supported by the Karanikas Co. Ltd. that provided the biological materials and extenders used in the study.

\section{References}

Boe-Hansen GB, Ersbøll AK, Greve T, Christensen P 2005: Increasing storage time of extended boar semen reduces sperm DNA integrity. Theriogenology 63: 2006-2019

De Ambrogi M, Ballester J, Saravia F, Caballero I, Johannisson A, Wallgren M, Andersson M, RodriguezMartinez H 2006: Effect of storage in short- and long-term commercial semen extenders on the motility, plasma membrane and chromatin integrity of boar spermatozoa. Int J Androl 29: 543-552

Estienne MJ, Harper AF, Day JL 2007: Characteristics of sperm motility in boar semen diluted in different extenders and stored for seven days at $18{ }^{\circ} \mathrm{C}$. Reprod Biology 7: 221-231

Foxcroft GR, Dyck MK, Ruiz-Sanchez A, Novac S, Dixon WT 2008: Identifying useable semen. Theriogenology 70: $1324-1336$

Fraser L, Strzezek J 2004: The use of comet assay to assess DNA integrity of boar spermatozoa following liquid preservation at $5{ }^{\circ} \mathrm{C}$ and $16{ }^{\circ} \mathrm{C}$. Folia Histochem Cyto 42: 49-55

Frydrychová S, Čeřovský J, Lustyková A, Rozkot M 2010: Effects of long-term liquid commercial semen extender and storage time on the membrane quality of boar semen. Czech J Anim Sci 55: 160-166

Gadea J 2003: Review: Semen extenders used in the artificial insemination of swine. Span J Agric Res 1: 17-27

Gadea J, Sellés E, Marco MA 2004: The predictive value of porcine seminal parameters on fertility outcome under commercial conditions. Reprod Domest Anim 39: 303-308

Haugan T, Gaustad AH, Reksen O, Grohn YT, Hofmo PO 2007: Fertility results of artificial inseminations performed with liquid boar semen stored in X-CellTM vs BTS extender. Reprod Domest Anim 42: 94-99

Kaeoket K, Srisowanna T, Wichaidit U, Chanapiwat P, Manee-in S 2010: Comparative study on six different long term commercial extenders for fresh boar semen. Thai J Vet Med 40: 257-263

Kaeoket K, Chanapai P, Junchiyaphoom P, Chanapiwat P 2011: The effect of using long term and short term extenders during cooling process on the quality of frozen boar semen. Thai J Vet Med 41: 283-288

Kommisrud E, Paulenz H, Sehested E, Grevle IS 2002: Influence of boar and semen parameters on motility and acrosome integrity in liquid boar semen stored for five days. Acta Vet Scand 43: 49-55

Kuster GE, Althouse GC 1999: The fecundity of porcine semen stored for 2 to 6 days in Androhep and X-cell extenders. Theriogenology 52: 365-376

Langendijk P, Soede NM, Kemp B 2005: Uterine activity, sperm transport, and the role of boar stimuli around insemination in sows. Theriogenology 63: 500-513

Maes D, Lopez RA, Rijsselaere T, Vyt P, Van Soom A 2011: Artificial insemination in pigs. In: Manafi M (Ed.): Artificial Insemination in Farm Animals. In Tech, Rijeka, pp. 79-94

Perez-Llano B, Enciso Garcia-Casado P, Sala R, Gosalvez J 2006: Sperm DNA fragmentation in boars is delayed or abolished by using sperm extenders. Theriogenology 66: 2137-2143

Rodríguez-Martínez H, Saravia F, Wallgrena M, Tienthai P, Johannisson A, Vázquez JM, Martínez E, Roca J, Sanze L, Calvete JJ 2005: Boar spermatozoa in the oviduct. Theriogenology 63: 514-535

Suarez SS, Pacey AA 2006: Sperm transport in the female reproductive tract. Hum Reprod Update 12: 23-37

Tardif S, Laforest JP, Cormier N, Bailey JL 1999: The importance of porcine sperm parameters on fertility in vivo. Theriogenology 52: 447-459

Tejada RI, Mitchell JC, Norman A, Marik JJ, Friedman S 1984: A test for the practical evaluation of male fertility by acridine orange (AO) fluorescence. Fertil Steril 42: 87-91

Tsakmakidis IA, Lymberopoulos AG, Khalifa TA 2010: Relationship between sperm quality traits and fieldfertility of porcine semen. J Vet Sci 11: 151-154

Vyt P, Maes D, Dejonckheere E, Castryck F, Van Soom A 2004: Comparative study on five different commercial extenders for boar semen. Reprod Domest Anim 39: 8-12 
Vyt P, Maes D, Quinten C, Rijsselaere T, Deley W, Aarts M, de Kruif A, Van Soom A 2008: Detailed motility evaluation of boar semen and its predictive value for reproductive performance in sows. Vlaams Diergen Tijds 77: 291-298

Willenburg KL, Miller GM, Rodriguez-Zas SL, Knox RV 2003: Effect of boar exposure at time of insemination on factors influencing fertility in gilts. J Anim Sci 81: 9-15

World Health Organization WHO 2010: Laboratory Manual for the Examination of Human Sperm and SemenCervical Mucus Interaction. WHO Press, Geneva 